\title{
Effect of Porcine Placenta Extract from Subcritical Water Extraction on Photodamage in Human Keratinocytes
}

\author{
Yooheon Park ${ }^{\dagger}$, Bok Kyung Han ${ }^{1 \dagger}$, Hyeon-Son $\mathrm{Choi}^{2}$, Yang Hee Hong ${ }^{3}$, \\ Eun Young Jung ${ }^{4}$, and Hyung Joo Suh* \\ Department of Food and Nutrition, Korea University, Seoul 136-703, Korea \\ ${ }^{1}$ BK Bio Co. Ltd. Sungnam 462-819, Korea \\ ${ }^{2}$ Department of Food Technology, Seoul Women's University, Seoul 139-774, Korea \\ ${ }^{3}$ Department of Beauty and Art, Suwon Women's University, Suwon 441-748, Korea \\ ${ }^{4}$ Department of Home Economic Education, Jeonju University, Jeonju 560-759, Korea
}

\begin{abstract}
The objective of this study was to evaluated the photoprotective effects of porcine placenta extract (PPE) on ultraviolet B (UVB)-induced oxidative stress in human keratinocytes ( $\mathrm{HaCaT}$ ) to evaluate its functional activities as a skin food ingredient. PPE prepared by subcritical water extraction was termed SPE, and subsequently digested by enzymes to prepare E-SPE. Increased intracellular reactive oxygen species (ROS) levels (192.0\%) induced by UVB were decreased by SPE and E-SPE. SPE had more effective ROS scavenging activity than E-SPE treatment. UVB treatment increased expression of tissue inhibitor of metalloproteinase 1 (TIMP-1), and this elevated expression was decreased by E-SPE treatment. High-dose treatment with E-SPE (50 and $100 \mu \mathrm{g} / \mathrm{mL})$ reduced TIMP-1 expression levels of UVB-C (control) to 33.5 and $34.6 \%$, respectively. In contrast, at low SPE doses ( 1 and $10 \mu \mathrm{g} / \mathrm{mL}$ ), the treatment slightly decreased TIMP1 expression levels to $73.3 \%$ and $71.3 \%$ of UVB-C, respectively. In conclusion, the present study demonstrated the protective effect of SPE and E-SPE against UVB damage in keratinocytes via ROS scavenging, down-regulating MMP-2 expression and up-regulating TIMP1 expression. This highlights the potential for SPE as an ingredient in the preparation of functional food against photoaging.
\end{abstract}

Key words: porcine placenta extract, subcritical water extraction, UVB, ROS, MMP-2, TIMP-1

Received November 3, 2014 / Revised November 23, 2014 / Accepted December 15, 2014

\section{Introduction}

Dairy products are a very commonly used food type in daily life. The huge demand on dairy products has led to the outgrowth of livestock. As the livestock business expands, concerns are being raised over the risk of environmental contamination by animal by-products, such as the placenta. Placenta extraction (PE) mix has been used in traditional Chinese medicines. The placenta contains and produces a wide variety of biologically active compounds, namely, proteins and peptides (Gude et al., 2004; Muralidhar and Panda, 1999). In addition, it is a rich source of hormones, cytokines, chemokines, and growth factors such as hepatocyte growth factor, epidermal growth

\footnotetext{
These authors equally contributed to this work.

*Corresponding author: Hyung Joo Suh, Department of Food and Nutrition, Korea University, Seoul 136-703, Korea. Tel.: +822-940-2853, Fax: +82-2-940-2859, E-mail: suh1960@korea.ac. $\mathrm{kr}$
}

factor, and transforming growth factor subtypes $\alpha$ and $\beta$ for which receptors have been identified in human placenta (Pal et al., 2002; Tonello et al., 1996).

In Chinese folk medicine, PE has been used for wound healing, and the medical uses and dermatological benefits of PEs have been reported. Recently, the benefits of topical application of PE on chronic and non-healing wounds have also been reported (Tiwary et al., 2006). Human PE also features as a component of various skin ointments and is used for skin revitalization, nourishment, melanocyte growth, and pigment-inducing effects (Pal et al., 2002). It is also used for the treatment of skin hypersensitivity conditions like dermatitis and psoriasis. Some typical human PEs contain keratinocyte growth factor (Failla et al., 2000; O'Keefe et al., 1985), or stimulators of endothelial cell growth (Presta et al., 1985).

It is interesting to note that animal-derived PEs are available commercially and regularly used in various ointments and lotions for the purpose of improving skin properties. Although the effects of human PE have been 
largely studied, only a few scientific studies report the effects of PE derived from animals such as pig, sheep, and horse. In rats with thermal injury, treatment with porcine PE (PPE) resulted in wound healing (Wu et al., 2003). However, the photoprotective effect of porcine $\mathrm{PE}$ on keratinocytes irradiated by ultraviolet B (UVB) has not been investigated.

Subcritical water extraction has been used successfully to selectively extract different classes of compounds. The process involves using hot water under pressure sufficient to maintain water in the liquid state. More polar compounds are extracted at lower temperatures, while less polar compounds are extracted at higher temperatures (Ibanez et al., 2003). One disadvantage associated with subcritical water extraction is the potential to inadvertently degrade the desired organic compounds during an extraction (Lindquist, 2011). Unexpected degradation of bioactive components can occur at the high temperatures and pressures using during subcritical water extraction. Therefore, the objective of this study was to evaluated the photoprotective effects of PPE on UVB-induced oxidative stress to evaluate its functional cosmetic activities as a skin ingredient.

\section{Materials and Methods}

\section{Preparation of PPE by subcritical water extraction}

Porcine placentas obtained following physiological delivery were washed thoroughly with sodium hydroxide $(\mathrm{NaOH})$ solution, cut into small pieces $\left(<1 \mathrm{~cm}^{2}\right)$, and rinsed in phosphate buffer solution as described previously (Georgieva et al., 1995). The tissue was then homogenized using a blender (HR-2084, Philips Electronics N.V., Netherlands) for $10 \mathrm{~min}$ and centrifuged. The precipitate was then mixed with water $(700 \mathrm{~mL})$ and extracted using a subcritical extractor (DIONEX ASE 100, Dionex Corporation, USA). During the extraction process, the pressure was maintained at 375 bar for $3 \mathrm{~h}$. Then, the extract was centrifuged at $2800 \mathrm{~g}$ for $20 \mathrm{~min}$. The supernatants were concentrated by using a vacuum evaporator at $40^{\circ} \mathrm{C}$ and lyophilized to produce PPE. Hereafter in the manuscript, PPE produced by the subcritical water extraction method will be referred to as SPE.

\section{Cell culture and UVB irradiation}

Human keratinocytes (HaCaT) cells were cultured in DMEM supplemented with 10\% FBS, 2 mM glutamine, and antibiotics. HepG2 cells were purchased from JCRB (Japan). Cells were incubated at $5 \% \mathrm{CO}_{2} / 95 \%$-humidified air at $37^{\circ} \mathrm{C}$. When the cells reached confluence, the medium was exchanged with phenol-free DMEM containing $2 \%$ fetal bovine solution. UVB irradiation was performed at $40 \mathrm{~mJ} / \mathrm{cm}^{2}(445 \mu \mathrm{W}$ for $1.5 \mathrm{~min})$ using a UVB lamp (GL20SE, Sankyo, Denki, Japan).

Cell cytotoxicity was determined by using a colorimetric MTT [3-(4,5-dimethylthiazol-2-yl)-2,5-diphenyltetrazolium bromide] assay to measure mitochondrial activity in viable cells using the method described by Mosmann (1983), but with minor modifications. Intracellular reactive oxygen species (ROS) was measured after $6 \mathrm{~h}$ of UVB irradiation, cell cytotoxicity after $24 \mathrm{~h}$, mRNA levels of matrix metalloproteinases (MMPs) after $12 \mathrm{~h}$, and protein levels of MMPs after $24 \mathrm{~h}$.

\section{Intracellular ROS}

$\mathrm{HaCaT}$ keratinocytes were placed in 24-well plates at a density of $2 \times 10^{5}$ cells per well for determination of ROS levels. After $6 \mathrm{~h}$ of UVB irradiation, the production of intracellular ROS was determined by DCFH-DA (2',7'dichlorofluorescin diacetate) assay (Shukla et al., 2006). DCFH-DA $(5 \mu \mathrm{g} / \mathrm{mL})$ was introduced to the cells, and DCFH-DA was detected at an excitation wavelength of $485 \mathrm{~nm}$ and an emission wavelength of $535 \mathrm{~nm}$ using a spectrofluorometer (VICTOR3TM, PerkinElmer, USA).

\section{Determination of mRNA level by semiquantitative} real time polymerase chain reaction (RT-PCR)

RNA isolation and RT-PCR analysis were performed as previously described (Ryu et al., 2009). Primer sequences used to amplify the desired cDNA were as follows: MMP2 forward and reverse primers: 5'-GGCCAAGTGGTCCGTGTG-3' and 5'-GAGGCCCCATAGAGCTCC-3'; MMP-9 forward and reverse primers: 5'-CACTGTCCAC CCCTCAGAGC-3' and 5'-GCCACTTGTCGGCGATA AGG-3'; HPRT forward and reverse primers: 5'-ATTCT TTGCTGACCTGCTGGATT-3' and 5'-CTTAGGCTTT GTATTTTGCTTTTC-3'.

\section{Statistical analysis}

Data are expressed as mean \pm standard deviation (SD). Statistical analysis was performed using the Statistical Package for Social Sciences (Version 12.0, SPSS Inc., USA). Unpaired Student's $t$-test and one-way analysis of variance (ANOVA) were used to assess statistical significance. For post hoc comparisons, one-sided Duncan's multiple range tests were used. Differences were considered statistically significant when $p<0.05$. 


\section{Results}

\section{SPE and E-SPE inhibit photodamage}

The cytotoxic effects of SPE and E-SPE were determined in a cell culture system. Serial concentrations of these extracts $(50-250 \mu \mathrm{g} / \mathrm{mL})$ were added to the medium and cells were incubated for $24 \mathrm{~h}$. As shown in Table 1, SPE was not associated with cytotoxicity at a concentration of $250 \mu \mathrm{g} / \mathrm{mL}$ in the absence of UVB treatment. However, cells survival rate was $93.1 \%$ under these conditions. HaCaT cells were exposed to UVB at an intensity of $40 \mathrm{~mJ} / \mathrm{cm}^{2}$, and cellular activity was detected for a following $24 \mathrm{~h}$. The survival rate of cells exposed to UVB irradiation was decreased to $91.2 \%$ and $92.6 \%$ in the absence of SPE and E-SPE, respectively. However, the addition of SPE and E-SPE partially improved cellular activity. The concentration of both extracts that proved to have a protective effect on cells ranged between 1 and $250 \mu \mathrm{g} / \mathrm{mL}$. Furthermore, the protective concentrations of SPE and E-SPE were significantly different. In light of these findings, $100 \mu \mathrm{g} / \mathrm{mL}$ was selected as the maximum concentration in order to study the effects of SPE and ESPE in HaCaT cells.

\section{Intracellular ROS scavenging effects}

The intracellular ROS scavenging effects of SPE and E-SPE were assessed in UVB-irradiated cells using the oxidant sensitive fluorescent probe, DCFH-DA. As shown in Fig. 1, UVB irradiation induced a $192.0 \%$ increase in intracellular ROS levels compared with control. SPE and E-SPE treatment lessened the effect of UVB on intracellular ROS levels. SPE treatment at concentrations greater than $10 \mu \mathrm{g} / \mathrm{mL}$ lowered ROS levels by $32.5 \%$ compared with UVB exposure alone. E-SPE treatment was shown to decrease intracellular ROS levels to a similar extent. However, more effective ROS scavenging activity was

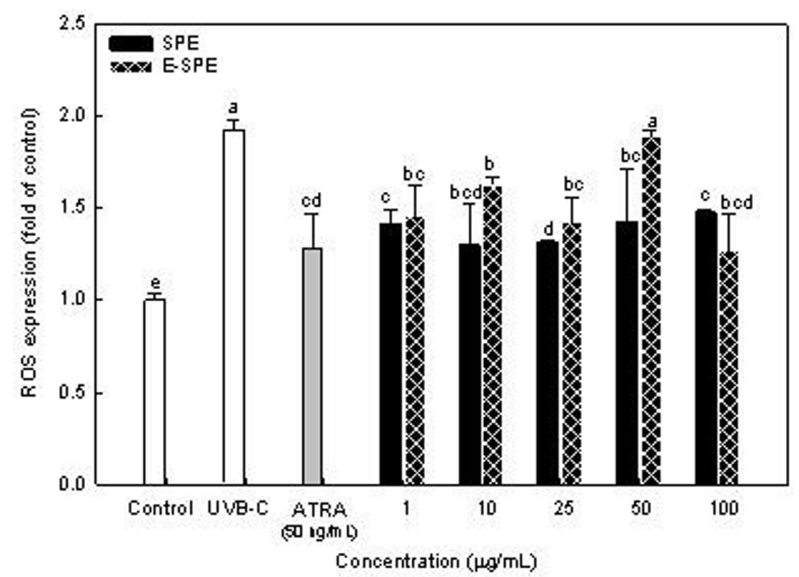

Fig. 1. Preventive effects of SPE and E-SPE on ROS generation in UVB-irradiated HaCaT cells. Data are expressed as \% of control without UVB irradiation. Values are expressed as mean $\pm \mathrm{SD}(\mathrm{n}=3)$. Different letters at each measurement indicate differences among groups $(p<0.05)$, and values were analyzed with repeated measures ANOVA. Control indicates control cells not exposed to UVB irradiation; UVB-C indicates cells exposed to UVB irradiation but not samples. All-trans retinoic acid (ATRA) was used as a positive control. E-SPE, porcine placental extract treated with digestive enzyme; HaCaT, human keratinocytes; ROS, reactive oxygen species; SPE: porcine placental extract prepared by subcritical water extraction; UVB, ultraviolet B; HaCaT, human keratinocytes.

demonstrated by SPE than E-SPE. In our previous study (Han et al., 2013), the SPE showed a noticeable activity in terms of antioxidant capacity for both measurement methods, FRAP and ABTS.

\section{Effects on MMP-2 expression}

MMP is the enzyme complex responsible for promoting degradation of skin membrane. Specifically, MMP-2 has gelatinase and collagenase activities. The mRNA levels and activity of MMP-2 were detected following 12-h

Table 1. Cell viability of SPE and E-SPE with or without UVB irradiation

\begin{tabular}{ccccc}
\hline \hline \multirow{2}{*}{ Conc. $(\mu \mathrm{g} / \mathrm{mL})$} & \multicolumn{3}{c}{ Cell viability (\% of normal control) } \\
\cline { 2 - 5 } & \multicolumn{2}{c}{ Control } & UVB irradiated \\
\cline { 2 - 5 } & SPE & E-SPE & $91.2 \pm 4.3^{\mathrm{a}}$ & $92.6 \pm 5.43^{\mathrm{a}}$ \\
1 & $100.0 \pm 3.2^{\mathrm{ns}}$ & $100.0 \pm 5.47^{\mathrm{ab}}$ & $96.7 \pm 0.3^{\mathrm{ab}}$ & $104.2 \pm 4.42^{\mathrm{ab}}$ \\
10 & $103.5 \pm 0.8$ & $104.0 \pm 7.08^{\mathrm{b}}$ & $98.0 \pm 3.5^{\mathrm{ab}}$ & $107.3 \pm 6.55^{\mathrm{b}}$ \\
50 & $106.1 \pm 1.9$ & $108.8 \pm 3.89^{\mathrm{b}}$ & $96.2 \pm 1.0^{\mathrm{ab}}$ & $106.7 \pm 4.61^{\mathrm{ab}}$ \\
100 & $105.1 \pm 1.8$ & $108.1 \pm 1.73^{\mathrm{b}}$ & $93.5 \pm 2.1^{\mathrm{ab}}$ & $106.9 \pm 5.92^{\mathrm{b}}$ \\
250 & $102.7 \pm 1.5$ & $102.3 \pm 4.98^{\mathrm{ab}}$ & $96.1 \pm 1.8^{\mathrm{ab}}$ & $97.8 \pm 3.42^{\mathrm{ab}}$ \\
\hline
\end{tabular}

Values are expressed as mean $\pm \mathrm{SD}(n=3)$. Different letters at each measurement indicate differences among groups $(p<0.05)$, and values were analyzed with repeated measures ANOVA. SPE: porcine placental extract prepared by subcritical water extraction; E-SPE, porcine placental extract treated with digestive enzyme; UVB, ultraviolet B. 

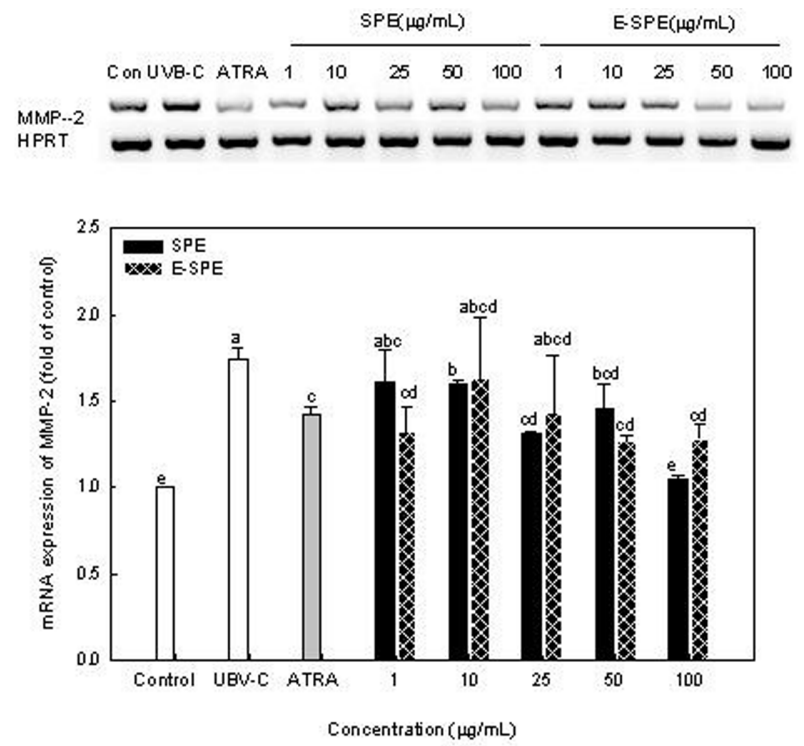

Fig. 2. Effect of SPE and E-SPE on MMP-2 mRNA expression. Values are expressed mean \pm SD $(n=3)$. Different letters at each measurement indicate differences among groups $(p<0.05)$, and values were analyzed with repeated measures ANOVA. ATRA, all-trans retinoic acid used as a positive control; Con, control; E-SPE: porcine placental extract treated with digestive enzyme; HPRT, hypoxanthine-guanine phosphoribosyltransferase; MMP-2, metalloproteinase-2; SPE, porcine placental extract prepared by subcritical water extraction; UVB-C, ultraviolet B control.

UVB irradiation of $\mathrm{HaCaT}$ cells at an intensity of $40 \mathrm{~mJ} /$ $\mathrm{cm}^{2}$. As shown in Fig. 2, MMP-2 activity was greater in the presence of UVB treatment than in the control group. However, further treatment of HaCaT cells with SPE or E-SPE significantly decreased MMP-2 activity in a dosedependent manner. Expression of MMP-2 in the group treated with $100 \mu \mathrm{g} / \mathrm{mL}$ SPE was similar to that of the control group. MMP-2 expression in the group treated with $100 \mu \mathrm{g} / \mathrm{mL}$ E-SPE was also slightly lower than the all-trans retinoic acid (ATRA, $50 \mathrm{ng} / \mathrm{mL}$ ) positive control group. And other expressions of MMPs (MMP-1, 9 and 13) were not showed significantly differences between control and the group treated with SPE and E-SPE (data were not shown). At high concentrations (50 and $100 \mu \mathrm{g} /$ $\mathrm{mL}$ ), SPE- and E-SPE-treated groups showed especially significant decreases in MMP-2 expression levels compared to the UVB control (UVB-C) group.

\section{Effects on tissue inhibitor of metalloproteinase expression}

Tissue inhibitor of metalloproteinase (TIMP) acts as an inhibitor of MMPs. Fig. 3 shows the effects of SPE and

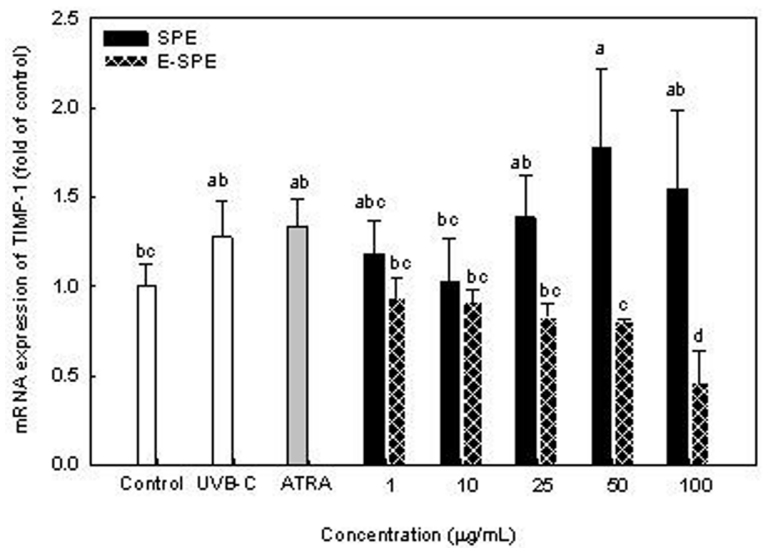

Fig. 3. Effect of SPE and E-SPE on TIMP-1 mRNA expression. Values are expressed mean \pm SD $(n=3)$. Different letters at each measurement indicate differences among groups $(p<0.05)$, and values were analyzed with repeated measures ANOVA. ATRA, all-trans retinoic acid used as a positive control; Con, control; E-SPE: porcine placental extract treated with digestive enzyme; SPE, porcine placental extract prepared by subcritical water extraction; TIMP-1, tissue inhibitor of metalloproteinase 1; UVB-C, ultraviolet B control.

E-SPE on TIMP-1 gene expression. UVB treatment increased expression of TIMP-1 compared with control, but its elevated expression was decreased by E-SPE treatment. High-dose treatment of E-SPE ( 50 and $100 \mu \mathrm{g} / \mathrm{mL}$ ) reduced TIMP-1 levels to $33.5 \%$ and $34.6 \%$, respectively, of UVB-C values. In contrast, low-dose SPE treatment (1 and $10 \mu \mathrm{g} / \mathrm{mL}$ ) slightly decreased TIMP-1 levels to $73.3 \%$ and $71.3 \%$ of UVB-C values, respectively. TIMP-1 mRNA expression was not influenced by high-dose treatments of SPE. TIMP-1 mRNA expression levels were generally lowered in the presence of SPE and E-SPE treatment, except for the high-dose E-SPE group. The positive effects of SPE and E-SPE on skin health are believed to be mediated through regulation of MMP-2 and TIMP-1 expression.

\section{Discussion}

Subcritical water extraction is a technique based on the use of water as an extractant, and uses temperatures between $100^{\circ} \mathrm{C}$ and $374^{\circ} \mathrm{C}$, and pressures high enough to maintain water in the liquid state. The technique is associated with greater selectivity, lower cost, shorter extraction times, and increased safety because it does not employ toxic organic solvents (Herrero et al., 2006; Ozel et al., 2003). A major advantage of using pressurized hot water for extraction is that the dielectric constant of water 
is reduced with increasing extraction temperatures, which decreases its polarity to facilitate the extraction of more nonpolar compounds (Rodríguez-Meizoso et al., 2006). In addition, the high pressures can further enhance the extraction of analytes trapped in matrix pores (GüçlüÜstündağ et al., 2007).

The critical point at which the water ionization constant increases is $374^{\circ} \mathrm{C}$ and $22 \mathrm{MPa}$; hence, water ionizes readily to hydrogen and hydroxide ions (Watchararuji et al., 2008). Hydrogen ions disrupt peptide bonds thereby resulting in hydrolysis of peptides (Brunner, 2009). Information on the effects of subcritical water for the hydrolysis of soybean and rice bran protein is available from previous reports (Sunphorka et al., 2012; Watchararuji et al., 2008). Although changes in the dielectric constant are often cited to justify this increase in solute solubility, loss of hydrogen-bonding capacity, polarizability, and cohesional energy density more closely reflect the molecular level changes occurring in water as its temperature is increased above boiling point (Srinivas et al., 2009). Thus, biologically active proteins or other large molecules in the placenta can be degraded or inactivated, and it is thought that these factors affect safety and biological activity.

SPE showed lower protein content than placenta homogenate, and was associated with decreasing antioxidant activity as the extraction time increased. Placenta homogenate and SPE demonstrated inhibitory effects on elastase activity; the half-maximal inhibitory concentration values were 46.1 and $34.7 \mu \mathrm{g} / \mathrm{mL}$, respectively. SPE has greater skin permeability properties than placenta homogenate (Han et al., 2013). PPE has also been shown to affect inflammatory responses (Jash et al., 2011). Han et al. (2013) also reported that PPE and its constituents regulate protein-energy malnutrition-induced fatigue through improving levels of immunity and decreasing fatiguerelated factors. PPE may have potential use as an agent for recovery from fatigue.

The placenta can be considered as a reservoir of cytokines, hormones, bioactive peptides, enzymes, growth factors, vitamins, and minerals (Togashi et al., 2002). PE also contains many valuable bioactive compounds that have various bio-capabilities such as inhibition of the following: aging, inflammation, sunburn, gene mutation, anaphylaxis, and oxidation (Kim et al., 2003).

Recently, benefits of the topical use of PE on chronic and non-healing wounds were reported (Tiwary et al., 2006). Intramuscular injection of human PE commercially known as "Placentrex" also healed radiation mucositis that developed in squamous cell carcinoma patients during radiation therapy (Kaushal et al., 2000). Human PE also features as a component of various skin ointments and is used for skin vitalizing, nourishment, melanocyte growth, and pigment-inducing activities (Pal et al., 2002), as well as for the treatment of skin hypersensitivity conditions, like dermatitis and psoriasis. However, the components and medicinal efficacy of PE have not been clarified in detail.

Ultraviolet (UV) radiation, in particular the middle wavelength (UVB, range 280-320 nm), elicits many adverse effects towards the skin, including cancer (Lyons and O'Brien, 2002), suppression of the immune system (Yoshikawa et al., 1990), and photoaging (Goihman-Yahr, 1996). UVB damage either directly or indirectly occurs through the production of ROS (Cunningham et al., 1985; Vile and Tyrrell, 1995). In the present study, UVB-irradiation induced an increase in intracellular ROS levels. However, SPE treatment in the presence of UVB irradiation significantly lowered ROS levels (Fig. 1).

ROS are produced as a molecular response of skin against UV exposure. However, over-exposure to UV rays leads to unregulated production of ROS, causing damage of cellular components such as lipids, protein, and DNA (Afaq et al., 2006). Particularly, UV-induced ROS production leads to DNA damage and subsequent apoptosis (Liu et al., 2007). ROS up-regulates the expression of MMPs, which mediate extracellular matrix degradation during pathological processes such as arthritis, inflammation, cardiovascular diseases, and cancer (Kong et al., 2010). Among them, MMP-2 (gelatinase A, $72 \mathrm{kDa}$ ) is capable of degrading basement membrane in the epidermis and are assumed to be involved in UV-mediated cutaneous damage (Park et al., 2006). High levels of MMPs are known to cause damage to skin connective tissue by degrading collagen and reducing collagen synthesis (Kong et al., 2010). We also demonstrated the increase of MMP2 expression by UVB irradiation. Therefore, the inhibitory effect of SPE and E-SPE against UVB damage was confirmed by its ability to block MMP-2 expression. In our previous study, SPE showed radical scavenging activity on ABTS and ferric reducing capacity (Han et al., 2013).

UVB exposure also affects the expression of TIMPs (Onoue et al., 2003). Among the identified TIMPs, TIMP1 and TIMP-2 are known as the major endogenous inhibitors that regulate the activity of MMP-2 (Corbel et al., 2000; Kong et al., 2010). The findings of the present study revealed that the presence of SPE essentially inhibited the effect mediated by UVB; this was achieved by suppress- 
ing the expression levels of MMP-2 and inhibiting the decreased expression of TIMP-1. As with SPE, E-SPE had a similar effect on ROS, MMP-2, and TIMP-1 expression. These findings suggest that SPE itself may become biologically active through gastric and intestinal digestion.

Although several types of placenta are commercially available, the use of human and bovine placenta is prohibited in cosmetics. In contrast, porcine placenta is relatively safe and its immune effect is similar to that of human placenta, therefore it is used widely in cosmetic agents. Indeed, porcine placenta has been shown to have beneficial skin care effects, to improve basic metabolic function, and strengthen immune function (Kim et al., 2011).

Notably, E-SPE showed a similar protective effect against UVB damage compared with SPE. Based on these properties, SPE is an effective natural component for preventing cellular damage induced by UVB exposure with potential dermatological and cosmetological value. SPE may provide benefits against photoaging when used as an ingredient for the preparation of functional cosmetic.

\section{Conflict of Interest}

The authors declare that there are no conflicts of interest.

\section{Acknowledgements}

This research was supported by Technology Development Program for Food, Ministry for Food, Agriculture, Forestry and Fisheries, Republic of Korea.

\section{References}

1. Afaq, F., Syed, D. N., Malik, A., Hadi, N., Sarfaraz, S., Kweon, M. H., Khan, N., Zaid, M. A., and Mukhtar, H. (2006) Delphinidin, an anthocyanidin in pigmented fruits and vegetables, protects human $\mathrm{HaCaT}$ keratinocytes and mouse skin against UVB-mediated oxidative stress and apoptosis. $J$. Invest. Dermatol. 127, 222-232.

2. Brunner, G. (2009) Near critical and supercritical water. Part I. Hydrolytic and hydrothermal processes. J. Supercrit. Fluids 47, 373-381.

3. Corbel, M., Boichot, E., and Lagente, V. (2000) Role of gelatinases MMP-2 and MMP-9 in tissue remodeling following acute lung injury. Braz. J. Med. Biol. Res. 33, 749-754.

4. Cunningham, M. L., Krinsky, N. I., Giovanazzi, S. M., and Peak, M. J. (1985) Superoxide anion is generated from cellular metabolites by solar radiation and its components. J. Free Radic. Biol. Med. 1, 381-385.
5. Failla, C. M., Odorisio, T., Cianfarani, F., Schietroma, C., Puddu, P., and Zambruno, G. (2000) Placenta growth factor is induced in human keratinocytes during wound healing. J. Invest. Dermatol. 115, 388-395.

6. Georgieva, R., Stefanov, D., Fichorova, R., and Dimitrova, E. (1995) Effects of the whole extract and the chromatographic fractions of the pig placenta on lymphocyte proliferation and humoral immune response. Theriogenology 44, 539-551.

7. Goihman-Yahr, M. (1996) Skin aging and photoaging: An outlook. Clin. Dermatol. 14, 153-160.

8. Gude, N. M., Roberts, C. T., Kalionis, B., and King, R. G. (2004). Growth and function of the normal human placenta. Thromb. Res. 114, 397-407.

9. Güçlü-Üstündă̆, Ö., Balsevich, J., and Mazza, G. (2007) Pressurized low polarity water extraction of saponins from cow cockle seed. J. Food Eng. 80, 619-630.

10. Han, J., Park, Y., and Suh, H. J. (2013) Skin permeability of porcine placenta extracts and its physiological activities. Korean J. Food Sci. An. 33, 356-362.

11. Herrero, M., Cifuentes, A., and Ibanez, E. (2006) Sub-and supercritical fluid extraction of functional ingredients from different natural sources: Plants, food-by-products, algae and microalgae: A review. Food Chem. 98, 136-148.

12. Ibanez, E., Kubatova, A., Senorans, F. J., Cavero, S., Reglero, G., and Hawthorne, S. B. (2003) Subcritical water extraction of antioxidant compounds from rosemary plants. J. Agric. Food Chem. 51, 375-382.

13. Jash, A., Kwon, H. K., Sahoo, A., Lee, C. G., So, J. S., Kim, J., Oh, Y. K., Kim, Y. B., and Im, S. H. (2011) Topical application of porcine placenta extract inhibits the progression of experimental contact hypersensitivity. J. Ethnopharmacol. 133, 654-662.

14. Kaushal, V., Verma, K., Manocha, S., Hooda, H., and Das, B. (2000) Clinical evaluation of human placental extract (placentrex) in radiation-induced oral mucositis. Int. J. Tiss. React. 23, 105-110.

15. Kim, H. J., Lee, J. W., Kim, Y. I., and Lee, M. H. (2003) The effect of placental extract on the expression of tyrosinase, TRP-1 and TRP-2 in SK30 melanoma cells. Korean J. Dermatol. 41, 1612-1618.

16. Kim, T., Kim, S., Kang, W., Baek, H., Jeon, H. Y., Kim, B. Y., Kim, C. G., and Kim, D. (2011) Porcine amniotic fluid as possible antiwrinkle cosmetic agent. Korean J. Chem. Eng. 28, 1839-1843.

17. Kong, C. S., Kim, J., Ahn, B., Byun, H. G., and Kim, S. K. (2010) Carboxymethylations of chitosan and chitin inhibit MMP expression and ROS scavenging in human fibrosarcoma cells. Process Biochem. 45, 179-186.

18. Lindquist, E. J. (2011) Stability of polycyclic aromatic hydrocarbons and benzoic acid derivatives under subcritical water conditions. East Carolina University.

19. Liu, S., Mizu, H., and Yamauchi, H. (2007) Molecular response to phototoxic stress of UVB-irradiated ketoprofen through arresting cell cycle in G2/M phase and inducing apoptosis. Biochem. Biophys. Res. Commun. 364, 650-655.

20. Lyons, N. M. and O'Brien, N. M. (2002) Modulatory effects 
of an algal extract containing astaxanthin on UVA-irradiated cells in culture. J. Dermatol. Sci. 30, 73-84.

21. Mosmann, T. (1983) Rapid colorimetric assay for cellular growth and survival: Application to proliferation and cytotoxicity assays. J. Immunol. Method 65, 55-63.

22. Muralidhar, R. and Panda, T. (1999) Useful products from human placenta. Bioprocess Eng. 20, 23-25.

23. O'Keefe, E. J., Payne, R. E., and Russell, N. (1985) Keratinocyte growth-promoting activity from human placenta. $J$. Cell. Physiol. 124, 439-445.

24. Onoue, S., Kobayashi, T., Takemoto, Y., Sasaki, I., and Shinkai, H. (2003) Induction of matrix metalloproteinase-9 secretion from human keratinocytes in culture by ultraviolet B irradiation. J. Dermatol. Sci. 33, 105-111.

25. Ozel, M. Z., Gogus, F., and Lewis, A. C. (2003) Subcritical water extraction of essential oils from Thymbra spicata. Food Chem. 82, 381-386.

26. Pal, P., Mallick, S., Mandal, S. K., Das, M., Dutta, A. K., Datta, P. K., Bera, R., and Bhadra, R. (2002) A human placental extract: in vivo and in vitro assessments of its melanocyte growth and pigment $\AA$ ]inducing activities. Int. J. Dermatol. 41, 760-767.

27. Park, C. H., Lee, M. J., Kim, J. P., Yoo, I. D., and Chung, J. H. (2006) Prevention of UV radiation-induced premature skin aging in hairless mice by the novel compound melanocin A. Photochem. Photobiol. 82, 574-578.

28. Presta, M., Mignatti, P., Mullins, D., and Moscatelli, D. (1985) Human placental tissue stimulates bovine capillary endothelial cell growth, migration and protease production. Biosci. Rep. 5, 783-790.

29. Rodríguez-Meizoso, I., Marin, F. R., Herrero, M., Señorans, F. J., Reglero, G., Cifuentes, A., and Ibáñez, E. (2006) Subcritical water extraction of nutraceuticals with antioxidant activity from oregano. Chemical and functional characterization. J. Pharm. Biomed. Anal. 41, 1560-1565.

30. Ryu, B., Li, Y., Qian, Z. J., Kim, M. M., and Kim, S. K. (2009) Differentiation of human osteosarcoma cells by isolated phlorotannins is subtly linked to COX-2, iNOS, MMPs, and MAPK signaling: Implication for chronic articular disease. Chem. Biol. Interact. 179, 192-201.
31. Shukla, S. K., Chaudhary, P., Prem Kumar, I., Afrin, F., Puri, S. C., Qazi, G. N., and Sharma, R.K. (2006) Cytotoxic and radioprotective effects of Podophyllum hexandrum. Environ. Toxicol. Pharmacol. 22, 113-120.

32. Srinivas, K., King, J., Monrad, J., Howard, L., and Hansen, C. (2009) Optimization of subcritical fluid extraction of bioactive compounds using Hansen solubility parameters. J. Food Sci. 74, E342-E354.

33. Sunphorka, S., Chavasiri, W., Oshima, Y., and Ngamprasertsith, S. (2012) Kinetic studies on rice bran protein hydrolysis in subcritical water. J. Supercrit. Fluids 65, 54-60.

34. Tiwary, S., Shukla, D., Tripathi, A., Agrawal, S., Singh, M., and Shukla, V. (2006) Effect of placental-extract gel and cream on non-healing wounds. J. Wound Care 15, 325.

35. Togashi, S. I., Takahashi, N., Iwama, M., Watanabe, S., Tamagawa, K., and Fukui, T. (2002) Antioxidative collagen-derived peptides in human-placenta extract. Placenta 23, 497502.

36. Tonello, G., Daglio, M., Zaccarelli, N., Sottofattori, E., Mazzei, M., and Balbi, A. (1996) Characterization and quantitation of the active polynucleotide fraction (PDRN) from human placenta, a tissue repair stimulating agent. J. Pharm. Biomed. Anal. 14, 1555-1560.

37. Vile, G. F. and Tyrrell, R. M. (1995) UVA radiation-induced oxidative damage to lipids and proteins in vitro and in human skin fibroblasts is dependent on iron and singlet oxygen. Free Radical Biol. Med. 18, 721-730.

38. Watchararuji, K., Goto, M., Sasaki, M., and Shotipruk, A. (2008) Value-added subcritical water hydrolysate from rice bran and soybean meal. Bioresour. Technol. 99, 6207-6213.

39. Wu, C. H., Chang, G.Y., Chang, W.C., Hsu, C. T., and Chen, R. S. (2003) Wound healing effects of porcine placental extracts on rats with thermal injury. Br. J. Dermatol. 148, 236245.

40. Yoshikawa, T., Rae, V., Bruins-Slot, W., van den Berg, J. W., Taylor, J. R., and Streilein, J. W. (1990) Susceptibility to effects of UVB radiation on induction of contact hypersensitivity as a risk factor for skin cancer in humans. J. Invest. Dermatol. 95, 530-536. 